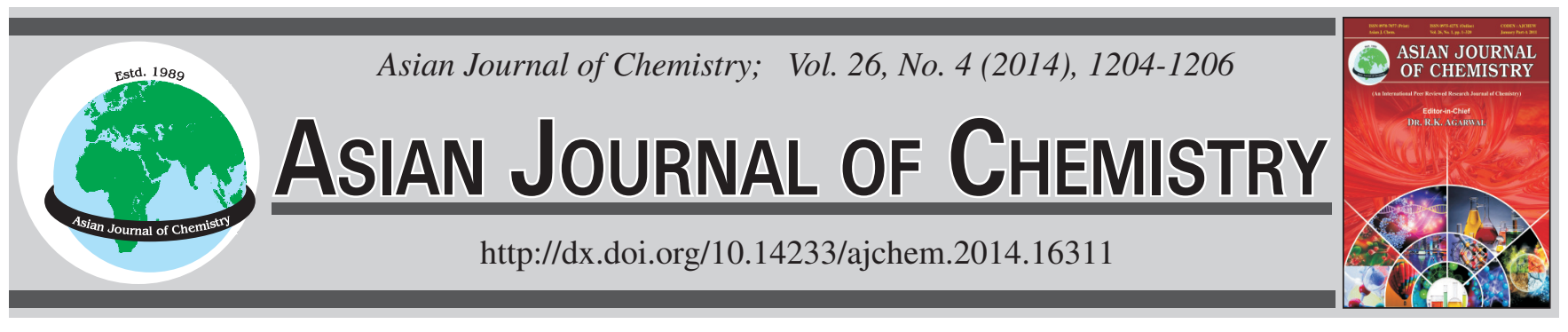

\title{
Synthesis and Characterization of Silver Nanoarticles from Extract of Eucalyptus citriodora
}

\author{
Muhammad Zahid QuReshi ${ }^{1}$, TABassum BashiR ${ }^{1, *}$, Shazia KhuRsheEd ${ }^{1}$, \\ Tarie Ismail ${ }^{1}$, Muhammad Ayub ${ }^{2}$, Alan Reynolds $^{3}$ and Ghulam Hussain ${ }^{1}$
}

${ }^{1}$ Department of Chemistry, Government College University, Lahore, Pakistan

${ }^{2}$ Department of Microbiology, Hazara University, Mansehra, Pakistan

${ }^{3}$ Experimental Techniques Center, Brunel University, London, UK

*Corresponding author: Tel: +92 333-4391934; E-mail: tabassumcaa@ gmail.com

The primary motivation for the study to develop simple eco-friendly green synthesis of silver nanoparticles using leaf extract of Eucalyptus citriodora as reducing and capping agent. The green synthesis process was quite fast and silver nanoparticles were formed within $0.5 \mathrm{~h}$. The synthesis of the particles was observed by UV-visible spectroscopy by noting increase in absorbance. Characterization of the particles was carried out by X-ray diffraction, FTIR and electron microscopy. The developed nanoparticles demonstrated that $E$. citriodora is good source of reducing agents. UV-visible absorption spectra of the reaction medium containing silver nanoparticles showed maximum absorbance at $460 \mathrm{~nm}$. FTIR analysis confirmed reduction of $\mathrm{Ag}^{+}$to $\mathrm{Ag}^{0}$ atom in silver nanoparticles. The XRD pattern revealed the crystalline structure of silver nanoparticles. The SEM analysis showed the size and shape of the nanoparticles. The method being green, fast, easy and cost effective can be recommended for large scale production of AgNPs for their use in food, medicine and materials.

Keywords: Silver nanoparticles, Eucalyptus citriodora, Capping agents, Scanning electron microscopy, Nanotechnology.

\section{INTRODUCTION}

Nanoparticles are being viewed as fundamental building blocks of nanotechnology. The optical, electrical, magnetic and catalytic properties of metal nanoparticles have been intensively studied during the last two decades because of their unique properties ${ }^{1}$. The development of biologically inspired experimental process for synthesis of nanoparticles is evolving into an important branch of nanotechnology $y^{2,3}$. Biogenic synthesis is useful not only because of its reduced environmental impact ${ }^{3,4}$ compared with some of the physiochemical production methods, but also because it can be used to produce large quantities of nanoparticles that are free of contamination and have a well define size and morphology ${ }^{5}$. Biosynthesis routes can actually provide nanoparticles of a better defined size and morphology than some of the physiochemical methods of production ${ }^{6}$. The antibacterial activities of silver nanoparticles are related to their size, with the smaller particles having higher activities on the basis of equivalent silver mass content ${ }^{7}$. Concerning the biological application of nanoparticles, it has been emphasized that the methods of synthesis through biological system. There are different plant extracts have been used and reported for synthesis of gold, silver and biometallic nanoparticles ${ }^{7}$.
In the present study, Eucalyptus citriodora was used for source of reducing agent. The plant is easily available in all the regions in Pakistan. Eucalyptus extract show various biological effects, such as antibacterial, antifungal, antihyperglycemic and antioxidant activities ${ }^{8}$. There are more than 500 Eucalyptus species, ranging from shrubs to several hundredfoot trees. Eucalyptus leaves and oil are utilized for medicinal and other uses, such as fragrance in perfumes. Volatile oils are derived principally from species that are rich in 1,8-cineol (eucalyptol, $\alpha$-monoterpene), such as Eucalyptus globulus Labillardiere (blue gum), E. smithii, or E. fructicetorum. E. globules Labillardiere is the most common medicinal species ${ }^{9}$.

\section{EXPERIMENTAL}

Preparation of plant extract: $10 \mathrm{~g}$ of fresh leaves of $E$. citridora were washed thoroughly with double-distilled water and were then cut into small pieces. These finely cut pieces were then mixed with $100 \mathrm{~mL}$ doubled distilled water and this mixture was kept for boiling for a period of $15 \mathrm{~min}$. After cooling, it was filtered through Whatman Filter paper No. 1. Filtrate placed at $4{ }^{\circ} \mathrm{C}$ for further experiment.

Synthesis of silver nanoparticles: $1 \mathrm{mM}$ aqueous solution of silver nitrate were prepared and used for the synthesis of 
silver nanoparticles. $10 \mathrm{~mL}$ of extract were taken and $100 \mathrm{~mL}$ of $\mathrm{AgNO}_{3}$ solution was added to it. The colour change from pale green to dark brown due to surface plasmon resonance. This occurs due to the collective oscillation of the conduction electrons confined to metallic nanoparticles. They were incubated at room temperature for $24 \mathrm{~h}$. The colour change indicate the synthesis of silver nanoparticles. UV-visible spectra showed strong SPR band at $460 \mathrm{~nm}$ and thus indicating the formation of silver nanoparticles The silver nanoparticles obtained by E. citriodora leaves extract were centrifuged at $13,000 \mathrm{rpm}$ for $25 \mathrm{~min}$ and subsequently dispersed in sterile distilled water to get rid of any uncoordinated biological materials.

UV-visible spectroscopy:UV-visible spectroscopic analysis was carried out on Shimadzu UV 1700. Cuvette of path length $10 \mathrm{~mm}$ was used. The measurements were carried out as a function of reaction time at room temperature.

X-ray diffractometry: XRD measurements were recorded on PANalyticalX'Pert PRO X-ray diffractometer. For XRD measurements, the silver nanoparticles were dried in oven at $60{ }^{\circ} \mathrm{C}$ and such dried powder was further analyzed on XRD for their phase structure and exact material identification. The $\mathrm{CuK}_{\alpha}$ radiation $(\lambda=1.582 \AA)$ was selected and the diffractogram was obtained in the $2 \theta$ range of $20-80^{\circ}$.

Fourier transform infrared (FTIR) spectroscopy: The binding properties of silver nanoparticles synthesized by $E$. citriodora leaf extract were investigated by FTIR analysis. FTIR measurements were taken on MIDAC 2000M series. Dried and powdered AgNPs were palleted with potassium bromide (KBr) (1:10 proportion). The spectra were recorded in the wavenumber range of $4000-450 \mathrm{~cm}^{-1}$ and analyzed by subtracting the spectrum of pure $\mathrm{KBr}$.

Scanning electron microscopic analysis: Scanning electron microscopic (SEM) analysis was done using JSM-6480 SEM machine. Thin films of synthesized and stabilized silver nanoparticles were prepared on a carbon coated copper grid by just dropping a very small amount of the sample on the grid and sample was analyzed for morphology and size of the silver nanoparticles.

\section{RESULTS AND DISCUSSION}

UV-visible spectroscopy: The formation of silver nanoparticles was observed upon the colour change of the leaf extract of Eucalyptus citriodora from transparent yellow into brown (Fig. 1), due to the coherentoscillation of electrons at the surface of nanoparticles, resulting insurface plasmon resonance ${ }^{9}$. The colour change into brown was noted within $20 \mathrm{~min}$ and the colour intensity increased significantly with increasing the $\mathrm{AgNO}_{3}$ concentration at a fixed volume of leaf extract of E. citriodora. The UV-visible spectrophotometry was also used to confirm the formation of the silver nanoparticles as shown in Fig. 1. From Fig. 1, the SPR band steadily increased in intensity with a prominent peak at about $460 \mathrm{~nm}$ at $1 \mathrm{mM}$ concentration. The change of colour and intensity of the SPR band might be due to the variation in concentration, size and shape of the resulting silver nanoparticles ${ }^{11}$.

XRD: The results of the XRD analysis showed $2 \theta$ intense values with various degree $\left(31.769^{\circ}, 37.605^{\circ}, 43.83^{\circ}, 64.07^{\circ}\right.$

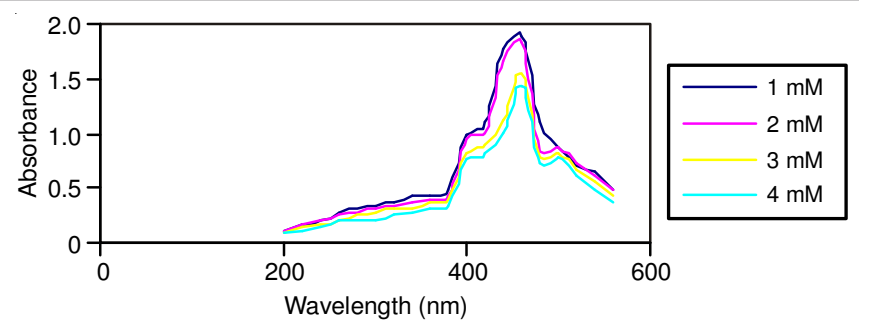

Fig. 1. UV-visible spectroscopy of silver nanoparticles

and $\left.77.20^{\circ}\right)$ these results are corresponds to (101), (111), (200), (220) and (311) Bragg's reflection based silver nanoparticles ${ }^{12}$ (Fig. 2).

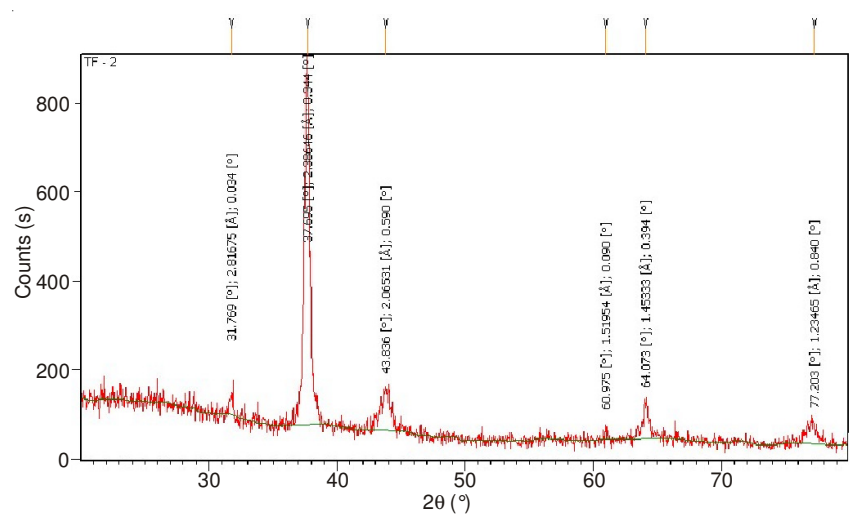

Fig. 2. XRD pattern of silver nanoparticle synthesis by leaf extract of $E$. citriodora

FTIR: The results of the FTIR used to identify the possible bio molecules responsible for the stabilization of the synthesized silver nanoparticles. The prominent peaks of the FTIR results are showing the correspond values to the alcohol, phenol group (O-H stretching-3424), amides group (N-H stretching3357), carboxylic group (O-H stretch-3280) alkenes, aromatics (C-H 3094), alkanes (C-H 2884), aliphatic saturated aldehydes $(\mathrm{C}=\mathrm{O}$ 1729), unsaturated aldehyde $(\mathrm{C}=\mathrm{O} 1667)$ and aromatic (C-C 1586). The observed peaks are considered as major functional groups in different chemical classes such as triterpenoids, flavonoids and polyphenols ${ }^{13}$. Hence, the terpenoids are proved to have good potential activity to convert the aldehyde groups to carboxylic acids in the metal ions. Further, amide groups are also responsible for the presence of the enzymes and these enzymes are responsible for the reduction synthesis and stabilization of the metal ions, further, polyphenols are also proved to have potential reducing agent in the synthesis of the silver nanoparticles ${ }^{13-15}$.

SEM: According to SEM analysis the silver nanoparticles were spherical in shape with varied particle size in $\mathrm{nm}$. The larger silver particles may be due to the aggregation of the smaller ones (Fig. 3).

\section{Conclusion}

The present study demonstrated the extracellular biosynthesis of an isotropic silver nanoparticles using the leaf extract of E. citriodora. We found that the leaves of E. citriodora can be a good source of synthesis of silver nanoparticles. The formation of silver nanoparticles was well studied. The silver nanoparticles characterization and morphology was studied 

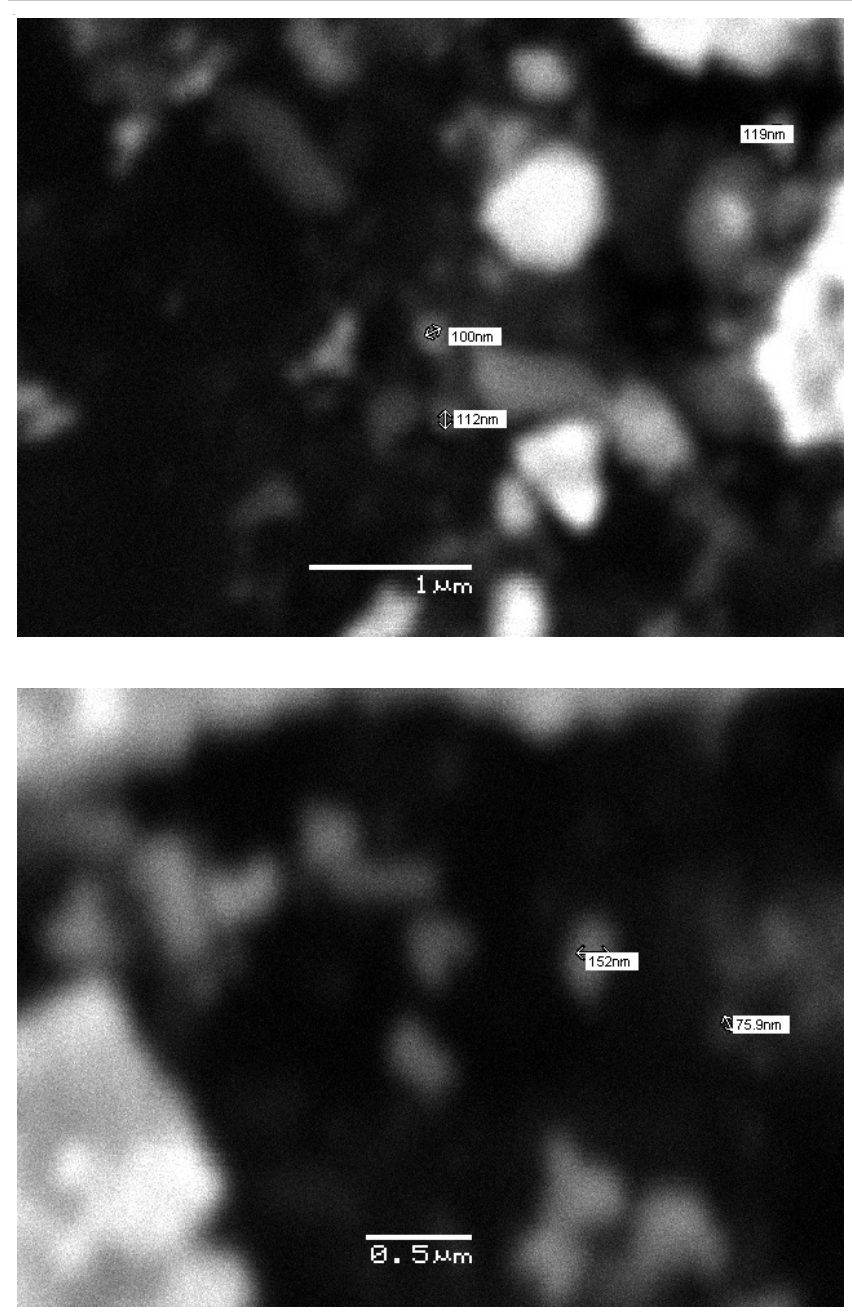

Fig. 3. SEM Micrograph of silver nanoparticles from E. citriodora with UV-visible spectroscopy, XRD and SEM techniques. The FTIR examination of the samples confirms the involvement of enzymes and amino groups in the reduction and stabilization of the silver nanoparticles. This procedure is easy, cost-effective, energy saving and environment friendly. It can scaled up for large scale synthesis of silver nanoparticles.

\section{REFERENCES}

1. H. Bar, D.K. Bhui, G.P. Sahoo, P. Sarkar, S.P. De and A. Misra, Colloids Surf. A, 339, 134 (2009).

2. A. Ahmad, P. Mukherjee, S. Senapati, D. Mandal, M.I. Khan, R. Kumar and M. Sastry, Colloids Surf. B, 28, 313 (2002).

3. S.S. Shankar, A. Rai, A. Ahmad and M. Sastry, J. Colloid Interf. Sci., 275, 496 (2004).

4. P.T. Anastas, J.B. Zimmerman, Why We Need a Green Nano Award \& How to Make it Happen, Woodrow Wilson International Center for Scholar, Washington, D.C. (2007).

5. J.E. Hutchison, ACS Nano, 2, 395 (2008)

6. P. Raveendran, J. Fu and S.L. Wallen, J. Am. Chem. Soc., 125, 13940 (2003).

7. K. Govindaraju, S.K. Basha, V.G. Kumar and G. Singaravelu, J. Mater. Sci., 43, 5115 (2008).

8. T. Takahashi, R. Kokubo and M. Sakaino, Lett. Appl. Microbiol., 39, 60 (2004).

9. K.B. Narayanan and N. Sakthivel, Mater. Res. Bull., 46, 1708 (2011).

10. P. Usha Rani and P. Rajasekharreddy, Colloids Surf. A, 389, 188 (2011).

11. M. Sathishkumar, K. Sneha, S.W. Won, C.-W. Cho, S. Kim and Y.-S. Yun, Colloids Surf. B, 73, 332 (2009).

12. A. Nabikhan, K. Kandasamy, A. Raj and N.M. Alikunhi, Colloids Surf. B, 79, 488 (2010).

13. T.N.V.K.V. Prasad and E.K. Elumalai, Trop. Biomed., 1, 439 (2011).

14. K.S. Mukunthan, E.K. Elumalai, T.N. Patel and V.R. Murty, Trop. Biomed., 1, 270 (2011).

15. S.N. Ngo, R.A. McKinnon and I. Stupans, Biochem. Physiol. C, Toxicol. Pharmacol., 136, 165 (2003). 